\title{
AVALIAÇÃO HISTOPATOLÓGICA DO FÍGADO DE BRACHYPLATYSTOMA ROUSSEAUXII (CASTELNAU, 1855) DA BAÍA DO GUAJARÁ, BELÉM, PARÁ
}

\author{
Rossineide Martins Rocha, ${ }^{1}$ Rodolpho Protázio Coelho, ${ }^{2}$ Caroline Silva Montes, ${ }^{2}$ \\ Simone Socorro Damasceno Santos ${ }^{2}$ e Maria Auxiliadora Pantoja Ferreira ${ }^{2}$ \\ 1. Professora adjunto III na Universidade Federal do Pará / E-mail: rmrocha@ufpa.br \\ 2. Universidade Federal do Pará.
}

RESUMO

\begin{abstract}
A espécie Brachyplatystoma rousseauxii constitui um dos recursos naturais de importância econômica para a região amazônica. O objetivo do presente trabalho foi analisar a histopatologia do fígado de $B$. rousseauxii como biomonitor da qualidade ambiental da Baía do Guajará. Capturaram-se os exemplares em quatro áreas distintas: uma área-controle e três áreas identificadas como locais de lançamentos de resíduos sólidos e líquidos, em quatro períodos: intermediário-chuvoso, chuvoso, intermediário-seco e seco. Coletaram-se 36 espécimes. Após a coleta, os peixes eram submetidos à biometria e os fragmentos do fígado processados para análise em microscopia de luz. Os animais coletados apresentaram
\end{abstract}

o comprimento médio total de $33,45 \mathrm{~cm}( \pm 7,72)$, peso total $239,21 \mathrm{~g}$ $( \pm 135,24)$ e peso hepático de $1,22 \mathrm{~g}( \pm 0,56)$. A análise histopatológica mostrou que $70 \%$ dos peixes apresentaram alterações hepáticas, sendo diagnosticadas como hepatite, inflamação, degeneração gordurosa e congestão e $30 \%$ apresentaram a estrutura hepática bem organizada, sendo considerados indivíduos sadios. De acordo com as alterações hepáticas observadas e o número de indivíduos coletados, sugere-se o comprometimento da Baía do Guajará e que a espécie $B$. rousseauxii provavelmente está adaptada às áreas onde foram registrados os lançamentos de poluidores ambientais.

PALAVRAS-CHAVES: Bioma amazônico, biomonitor, fígado, histopatologia, peixe.

ABSTRACT

\section{HISTOPATHOLOGICAL EVALUATION OF THE LIVER OF \\ BRACHYPLATYSTOMA ROUSSEAUXII (CASTELNAU, 1855) FROM GUAJARA'S BAY, BELÉM, PA}

The species Brachyplatystoma rousseauxii is one of the most important economic resources of the Amazon region. The objective of the present work was to analyze the histopathology of the liver of B. rousseauxii and to indicate this species as a biomonitor of the environmental quality of the Guajará Bay. Samples were collected in four areas: a control area and three areas identified as discharge sites of solid and liquid wastes. Sampling was carried out in four season periods: dry/wet season, wet season, wet/dry season and dry season. The number of collected specimens was 36 . The fishes were submitted to biometry after catching. They presented mean total length of $33.45 \mathrm{~cm}( \pm 7.72)$, total weight of $239.21 \mathrm{~g}$ $( \pm 135.24)$, and liver weight of $1.22 \mathrm{~g}( \pm 0.56)$. Liver fragments were processed for light microscopy analysis. Histopathologic analysis demonstrated that $70 \%$ of the fish presented hepatitis, inflammation, fatty degeneration, and congestion, and that $30 \%$ presented well-organized liver structures, and they were considered healthy. The hepatic changes observed and the number of specimens collected suggest that the environmental quality of the Guajará Bay is affected and that the species $B$. rousseauxii is probably adapted to the areas where there are waste discharges.

KEY WORDS: Amazonian biome, biomonitor, liver, histopathology, fish. 


\section{INTRODUÇÃO}

A espécie Brachyplatystoma rousseauxii, vulgarmente conhecida como dourada, constitui um dos recursos pesqueiros mais importantes no baixo Amazonas. É considerada um dos itens mais importantes de exportação, contribuindo significativamente para a economia local (RUFFINO \& ISAAC, 2000, PETRERE et al., 2004; RUFFINO, 2005).

Os peixes são usualmente considerados como bons biomonitores para avaliação dos efeitos da poluição ambiental, por estarem localizados no topo da cadeia trófica aquática e por acumularem substâncias tóxicas (GERNHÖFER et al., 2001). A baixa qualidade da água pode ter consequência danosa às populações piscívoras (WINKALER et al., 2001) e à população humana que utiliza esse recurso como fonte de subsistência (RUFFINO, 2002; BARTHEM \& FABRÉ, 2004).

O uso de biomonitores para estudos histopatológicos é feito com a finalidade de avaliar o impacto que os organismos aquáticos sofrem em nível corpóreo, seja tecidual, bioquímico, fisiológico, comportamental ou energético em consequência da exposição do animal às substâncias químicas do ambiente aquático (LIVINGSTONE, 1993; GERNHÖFER et al., 2001; WINKALER et al., 2001; DE LA TORRE et al., 2005).

Nos peixes, os estudos histopatológicos são direcionados para diferentes órgãos, em especial os responsáveis pelo metabolismo de substâncias tóxicas, como o fígado, que pode sofrer alteração estrutural e metabólica mediante a exposição de poluentes, a alimentação, as toxinas, os parasitos e os micro-organismos (ARELLANO et al., 1999; GERNHÖFER et al., 2001; FANTA et al., 2003; AU, 2004; BOMBONATO et al., 2007). Essas alterações podem refletir em uma variedade de processos patológicos com possibilidade de levar até a morte do animal (HIBIYA, 1982).

O fígado dos peixes teleósteos, em geral, é constituído por três lobos (BRUSLÉ \& ANADON, 1996). No entanto, as particularidades quanto ao arranjo e disposição dos lobos variam entre diferentes espécies e até mesmo em indivíduos da mesma espécie (BOMBONATO et al., 2007). Microscopicamente, o fígado é formado por placas celulares circundadas por sinusoides em torno da veia centro lobular, e cada placa apresenta hepatócitos com uma face sinusoidal para absorção e uma face biliar para excreção. No parênquima hepático são observadas células melanomacrófagos, localizadas próximas às regiões portais que se organizam formando o centro melanomacrófago (BRUSLÉ \& ANADON, 1996). Estes centros atuam como elementos do sistema imune (HARTLEY et al., 1996). Em torno da veia portal, ainda podem ser observadas células de tecido pancreático, as quais apresentam arranjo acinar (BRUSLÉ \& ANADON, 1996). A combinação do tecido hepático e pancreático caracteriza o hepatopâncreas (HIBIYA, 1982; FIGUEIREDO-FERNANDES et al., 2007a).O objetivo do presente trabalho é analisar a histopatologia do fígado de $B$. rousseauxii (CASTELNAU, 1855) como biomonitor da qualidade ambiental da baía do Guajará, Belém, PA.

\section{MATERIAL E MÉTODOS}

Realizaram-se capturas de $B$. rousseauxii em quatro áreas na baía do Guajará (Belém, PA): área I (AI) - controle, ausente de fontes poluidoras; área II (AII) - caracterizada por dejetos de indústria pesqueira e esgoto doméstico; área III (AIII) - presença de dejetos domésticos; área IV (AIV) - dejetos de embarque e desembarque de navios de médio porte, de desembarque pesqueiro e lixo doméstico. As coletas foram realizadas em quatro períodos: intermediário chuvoso (dezembro/2004); chuvoso (março/2005); intermediário seco (junho/2005) e seco (setembro/2005).

Após a captura, os exemplares eram identificados, acondicionados em caixa isotérmica contendo gelo para impedir a autólise do tecido e imediatamente transportados ao laboratório para realização da biometria (peso e comprimento). Em seguida os peixes eram submetidos a uma incisão ventral do poro urogenital até o opérculo, para facilitar a retirada das vísceras. $\mathrm{O}$ fígado era separado, pesado e os fragmentos desse órgão eram fixados em solução de Bouin por vinte e quatro horas.

Após a fixação, as amostras foram desidratadas em concentrações crescentes de etanol, desde $50 \%$ até etanol absoluto, diafanizadas em xilol, infiltradas e incluídas em parafina. Em seguida, obtiveram-se cortes transversais de $5 \mu \mathrm{m}$ de espessura, sendo corados com hematoxilina-eosina, analisados e fotografados em microscópico Olympus CH30. 
A análise estatística foi feita utilizando-se análise de variância e teste de Bonferroni com auxílio do software Bioestat 5.0 (AYRES et al., 2007).

\section{RESULTADOS}

Durante o período de estudo, capturou-se um total de 36 espécimes de $B$. rousseauxii: dez indivíduos
= intermediário-chuvoso; cinco indivíduos = chuvoso; 16 indivíduos $=$ intermediário-seco e cinco indivíduos $=$ seco. $\mathrm{Na}$ área I (controle), foi capturado o maior número de exemplares. Nesta, observaram-se indivíduos com fígados normais e em processos patológicos em proporções iguais. Já a área II apresentou maior número de indivíduos com fígados alterados (Tabela 1).

TABELA 1. Número de indivíduos capturados nas áreas de estudo em relação ao período de estudo e análise histopatológica do fígado

\begin{tabular}{|c|c|c|c|c|c|c|c|c|c|c|}
\hline \multirow{2}{*}{$\begin{array}{l}\text { Períodos } \\
\text { Área de estudo }\end{array}$} & \multicolumn{2}{|c|}{$\begin{array}{l}\text { Intermediário- } \\
\text { chuvoso }\end{array}$} & \multicolumn{2}{|c|}{ Chuvoso } & \multicolumn{2}{|c|}{ Intermediário-seco } & \multicolumn{2}{|l|}{ Seco } & \multicolumn{2}{|c|}{ Total } \\
\hline & $\mathrm{N}$ & A & $\mathrm{N}$ & A & $\mathrm{N}$ & A & $\mathrm{N}$ & A & $\mathrm{N}$ & A \\
\hline AI & 0 & 1 & 2 & 0 & 5 & 5 & 1 & 2 & 8 & 8 \\
\hline AII & 2 & 7 & 0 & 2 & 0 & 0 & 0 & 2 & 2 & 11 \\
\hline AIII & 0 & 0 & 0 & 0 & 1 & 5 & 0 & 0 & 1 & 5 \\
\hline AIV & 0 & 0 & 0 & 1 & 0 & 0 & 0 & 0 & 0 & 1 \\
\hline Total & \multicolumn{2}{|c|}{10} & \multicolumn{2}{|c|}{5} & \multicolumn{2}{|c|}{16} & \multicolumn{2}{|c|}{5} & \multicolumn{2}{|c|}{36} \\
\hline
\end{tabular}

$\mathrm{N}=\mathrm{n}^{\circ}$ de peixes com fígado normal $/ \mathrm{A}=\mathrm{n}^{\circ}$ de peixes com fígado alterado, $\mathrm{AI}=$ área $\mathrm{I}$ (controle), $\mathrm{AII}=$ área $\mathrm{II}$ (presença de dejetos industriais e domésticos), AIII= área III (presença de dejetos domésticos), AIV = área IV (presença de dejetos domésticos e de navios).

A análise das medidas de peso total, comprimento total e peso hepático entre espécimes normais e alterados está descrita na Tabela 2. A área IV não foi considerada, em virtude da captura de um único exemplar.

As médias de comprimento total, de peso total e de peso do fígado de peixes com alterações hepática das três áreas da baía do Guajará comparada com a média de peixes normais estão descritas na Tabela 3.
TABELA 2. Médias ( $\pm \mathrm{DP})$ de comprimento, peso total e peso do fígado de $B$. rousseauxii nas três áreas de estudo da baía do Guajará

\begin{tabular}{lccc}
\hline $\begin{array}{l}\text { Área de estudo/ } \\
\text { Biometria }\end{array}$ & $\begin{array}{c}\text { AI } \\
(\mathrm{n}=16)\end{array}$ & $\begin{array}{c}\text { AII } \\
(\mathrm{n}=13)\end{array}$ & $\begin{array}{c}\text { AIII } \\
(\mathrm{n}=6)\end{array}$ \\
\hline Comprimento & $30,39( \pm 7,21)^{\mathrm{a}}$ & $34,05( \pm 6,58)^{\mathrm{ab}}$ & $25,51( \pm 1,89)^{\mathrm{ac}}$ \\
Peso total & $171,13( \pm 138,2)^{\mathrm{a}}$ & $242,72( \pm 128,43)^{\mathrm{a}}$ & $79,5( \pm 34,23)^{\mathrm{b}}$ \\
Peso hepático & $0,91( \pm 0,78)^{\mathrm{a}}$ & $1,21( \pm 0,51)^{\mathrm{a}}$ & $0,4( \pm 0,3)^{\mathrm{b}}$ \\
\hline
\end{tabular}

*Sobrescritos distintos na mesma linha indicam diferença estatística $(\mathrm{P}<0,01) . \mathrm{n}=\mathrm{n}^{\circ}$ de peixes por área de estudo, $\mathrm{AI}=$ área I (controle), AII = área II (presença de dejetos industriais e domésticos), AIII= área III (presença de dejetos domésticos).

TABELA 3. Médias $( \pm \mathrm{DP})$ de peso total e de peso do fígado de $B$. rousseauxii com alteração hepática em três áreas de estudo da baía do Guajará

\begin{tabular}{lcccc}
\hline & AI & AII & AIII \\
\hline $\begin{array}{c}\text { Área de estudo/ } \\
\text { biometria }\end{array}$ & $\begin{array}{c}\text { Normal } \\
(\mathrm{n}=8)\end{array}$ & $\begin{array}{c}\text { Alterado } \\
(\mathrm{n}=8)\end{array}$ & $\begin{array}{c}\text { Alterado } \\
(\mathrm{n}=11)\end{array}$ & $\begin{array}{c}\text { Alterado } \\
(\mathrm{n}=5)\end{array}$ \\
\hline Peso total & $178,69( \pm 162,08)^{\mathrm{a}}$ & $163,56( \pm 120,52)^{\mathrm{ab}}$ & $239,21( \pm 135,24)^{\mathrm{a}}$ & $3,82( \pm 36,4)^{\mathrm{b}}$ \\
Peso hepático & $0,81( \pm 0,7)^{\mathrm{c}}$ & $1,02( \pm 0,84)^{\mathrm{a}}$ & $1,22( \pm 0,56)^{\mathrm{a}}$ & $0,48( \pm 0,34)^{\mathrm{b}}$ \\
\hline
\end{tabular}

*Sobrescritos distintos na mesma linha indicam diferença estatística $(\mathrm{P}<0,025)$; normal $\mathrm{n}=\mathrm{n}^{\circ}$ de peixes com fígado normal por área de estudo; alterado $\mathrm{n}=\mathrm{n}^{\circ}$ de peixes com fígado alterado por área de estudo; $\mathrm{AI}=$ área $\mathrm{I}$ (controle); $\mathrm{AII}$ = área II (presença de dejetos industriais e domésticos); $\mathrm{AIII}=$ área III (presença de dejetos domésticos). 
Macroscopicamente, o fígado normal apresentou uma estrutura homogênea, com coloração marromavermelhada, em virtude de sua rica vascularização, localizado na região medial e caudalmente ao coração, posterior às brânquias. $\mathrm{O}$ órgão apresentou-se dividido em dois lobos: um maior-anterior (1A) e outro menorposterior de formato piramidal localizado próximo ao hilo (1B).
Histologicamente, o parênquima hepático era constituído pela veia centro lobular (VCL) circundado por cordões de hepatócitos e por capilares sinusoides. O hepatócito apresentou formato poligonal com núcleo esférico e centralizado (Figura 2A). Ainda foram observados próximo às veias e artérias hepáticas tecido pancreático exócrino e centros melanomacrófagos. Este último apresentou coloração acastanhada (Figura 2B).
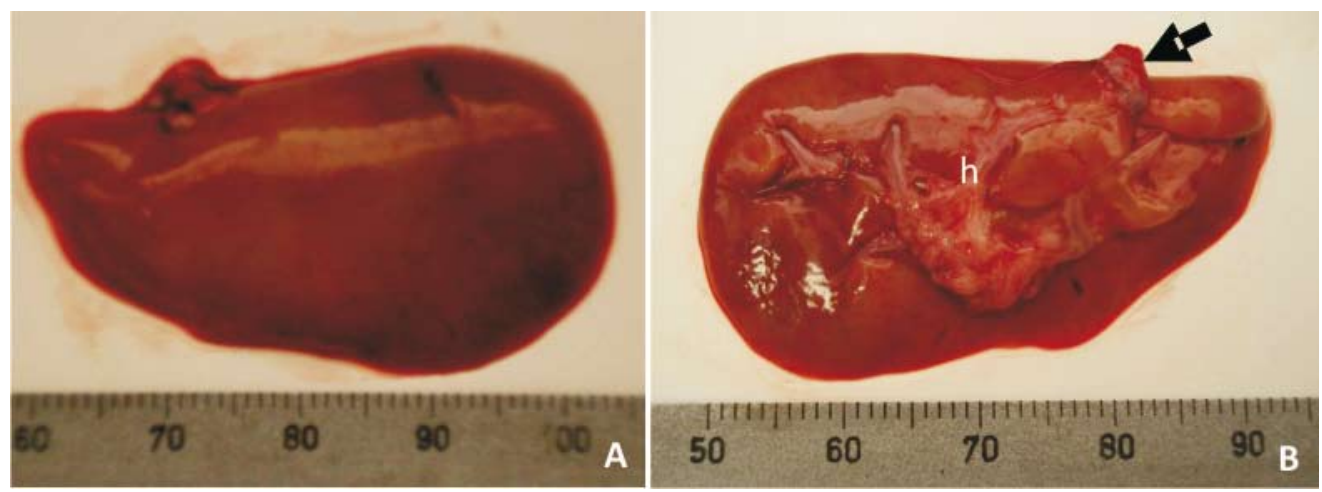

FIGURA 1. Fotografia do fígado normal: A - visão dorsal. B - visão ventral. Observar o hilo (h) e o lóbulo (seta).
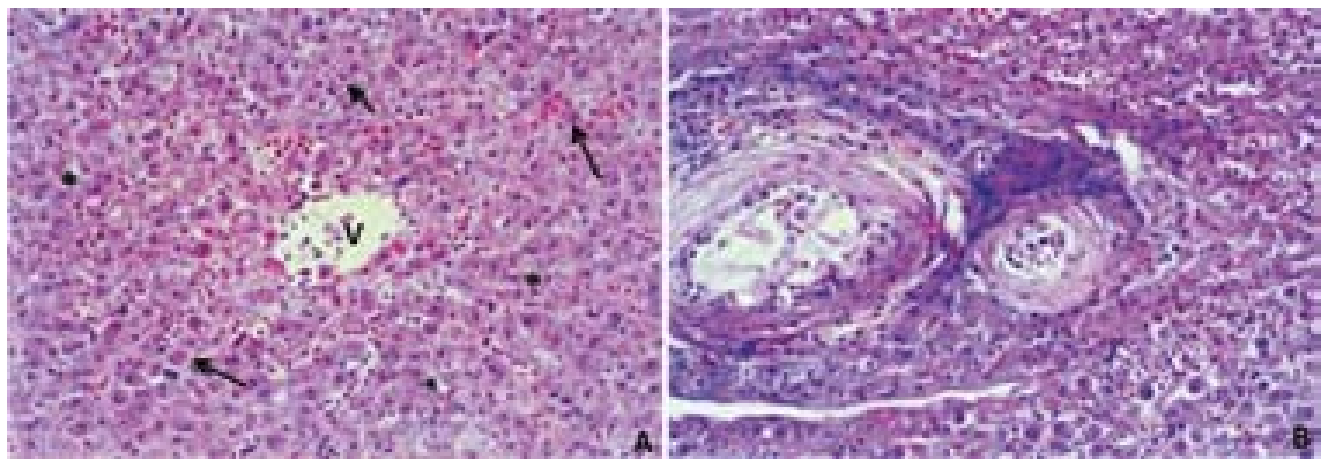

FIGURA 2. Fotomicrografia do fígado normal: A - parênquima hepático com VCL (V), cordões de hepatócitos (seta fina) e capilares sinusoides $(*)$. HE, objetiva 40x; B - artérias hepáticas (a), centros melanomacrófagos (c) e tecido pancreático exócrino (p). HE, objetiva 40x.

A análise microscópica mostrou que $70 \%$ dos exemplares de $B$. rousseauxi apresentaram as seguintes alterações hepáticas: hepatite, inflamação, degeneração gordurosa e congestão.

Nos fígados com hepatite, o parênquima estava desorganizado, a VCL encontrava-se dilatada, os hepatócitos apresentavam-se hipertrofiados com perda da integridade citoplasmática, o núcleo era visível e pouco centralizado (Figura 3A). Os sinusoides hepáticos foram pouco definidos, porém em algumas áreas havia concentração de células sanguíneas (Figura 3B).

Nos fígados com inflamação, o parênquima apresentou um aspecto esponjoso e em algumas áreas havia depósito de lipídio (Figura 4A). Os hepatócitos estavam aumentados e vacuolizados, com deslocamento do núcleo para a periferia da célula, observando-se infiltrados leucocitários próximos às células hepáticas (Figura 4B).

Os fígados com degeneração gordurosa caracterizaram-se pelo aparecimento de gotículas de gordura 
isoladas ou agrupadas. Os hepatócitos apresentaram aspecto normal e os sinusoides distinguíveis (Figura 5A). Em geral, estas estruturas encontraram-se próximas aos vasos sanguíneos (Figura 5B).

Nos fígados com congestão, o parênquima apresentou um infiltrado leucocitário ao redor dos vasos sanguíneos, sendo possível observar centros melanomacrófagos em algumas áreas (Figura 6A). Os hepatócitos apresentaram aspecto normal e os capilares sinusoides mostraram-se bem delimitados e as veias dilatadas. Nesses fígados, foram observadas células pancreáticas em torno de arteríola, vênula e do ducto biliar (Figura 6B).
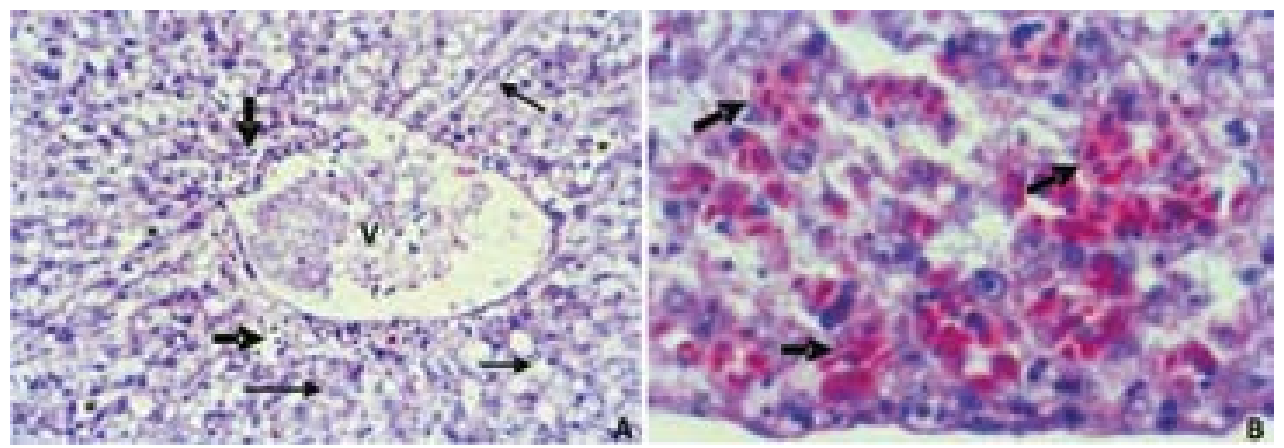

FIGURA 3. Fotomicrografia do fígado com hepatite: A- VCL dilatadas (V), hepatócitos hipertrofiados (seta fina), capilares sinusoides (*) e células sanguíneas (seta grossa). HE, objetiva 40x; B- Detalhe do acúmulo de células sanguíneas (seta grossa) no parênquima hepático. HE, objetiva 100x.
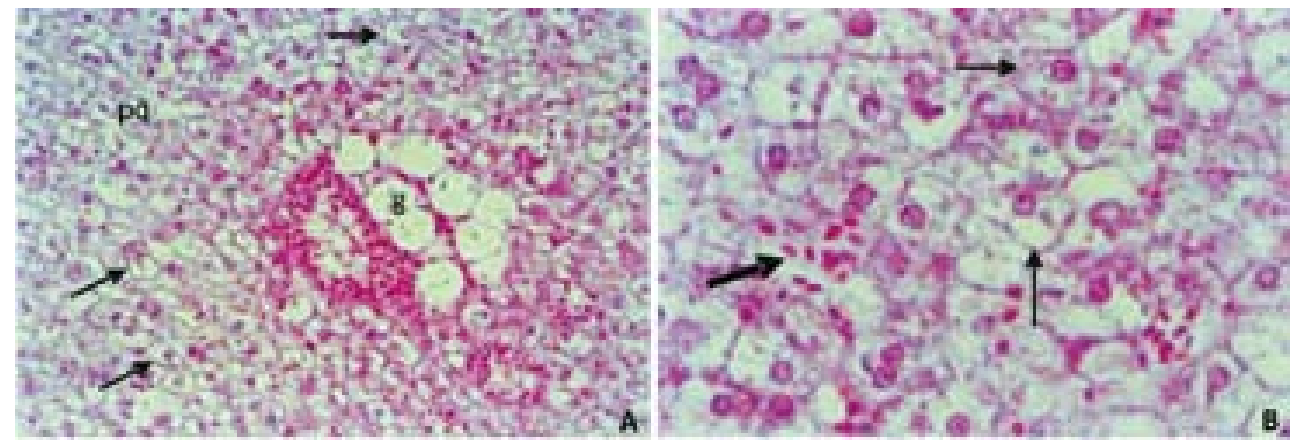

FIGURA 4. Fotomicrografia do fígado com inflamação: A - parênquima (pq) com aspecto esponjoso, hepatócito vacuolizado (seta fina), depósito de lipídio (g). HE, objetiva 40x. B - Detalhe dos hepatócitos vacuolizados (seta fina) e algumas células sanguíneas (seta grossa). HE, objetiva 100x.
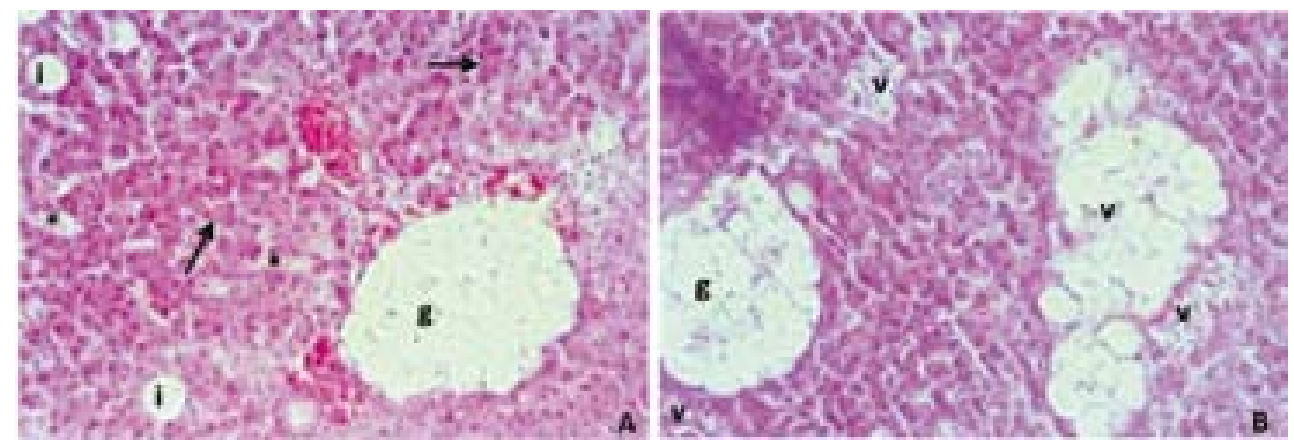

FIGURA 5. Fotomicrografia do fígado com degeneração gordurosa: A - parênquima com gotículas de gordura isoladas (i) e agrupadas (g), sinusoides (*) e cordões de hepatócitos (seta fina). HE, objetiva 40x; B - Gotículas de gordura agrupadas próximas ao vaso sanguíneo (v). HE, objetiva 40x. 


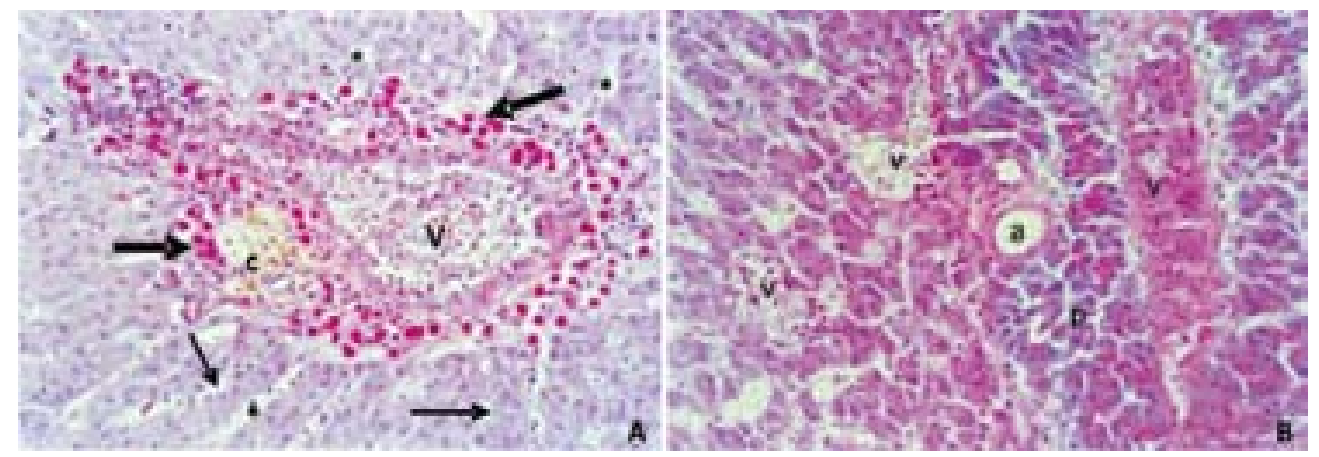

FIGURA 6. Fotomicrografia do fígado com congestão: A - Extravasamento de células sanguíneas (seta grossa) ao redor da VCL (V), presença de centro melanomacrófago (c), cordões (seta fina) e sinusoides hepáticos (*) distintos. HE, objetiva 40x. B - Presença de tecido pancreático exócrino (p), artéria (a) e veias dilatadas (v). HE, objetiva 40x.

\section{DISCUSSÃO}

O fígado de $B$. rousseauxii apresentou características anatômicas similares a outros teleósteos (BRUSLÉ \& ANADON, 1996; VICENTINI et al., 2005). O estudo histológico do fígado dessa espécie revelou que o órgão não apresenta divisões em lóbulos, sendo impossível distinguir subdivisões hexagonais, resultados similares aos descritos em Leporinus macrocephalus (BOMBONATO et al., 2007).

$\mathrm{Na}$ espécie em estudo, foi possível observar a tríade portal, constituída pela arteríola, vênula e ducto biliar, sendo encontrada com menor frequência. Segundo BRUSLÉ \& ANADON (1996), as tríades portais são indistintas, se não ausentes em quase todos os teleósteos. Em algumas estruturas portais de B. rousseauxii, observaram-se células do tecido pancreático exócrino em torno dos vasos. De acordo com FIGUEIREDOFERNANDES et al. (2007a), é frequente a presença dos pancreócitos no fígado de Oreochromis niloticus caracterizando um arranjo hepatopancreático. $\mathrm{O}$ desenvolvimento do tecido pancreático exócrino em torno dos vasos acontece durante a ontogênese, e este tecido pode permanecer extra-hepático ou penetrar no fígado (BRUSLÉ \& ANADON, 1996; BOMBONATO et al., 2007).

$\mathrm{Na}$ área I (controle), capturou-se o maior número de espécimes. Neste local, observaram-se cardumes de pequenos peixes da família Engraulidae, que é a base da cadeia alimentar de B. rousseauxii (BARBO$\mathrm{SA}, 2005)$. As demais áreas apresentaram reduzido número de peixes. Essas áreas foram diagnosticadas como impactadas, visto que apresentam um acelerado processo de ocupação populacional e industrial da cidade de Belém, sendo evidenciada uma modificação ambiental com redução do número de espécies de vida aquática e presença de dejetos domésticos e industriais (PINHEIRO, 1987; COHAB, 1997). Isto sem contar a presença de coliformes totais e fecais acima do limite estabelecido pela Portaria do Conama n. ${ }^{\circ} 20 / 86$, tratando-se de águas pertencentes à classe II (SILVA, 2006).

Em relação aos períodos de captura, foi observado que nos períodos chuvoso e seco houve uma redução do número de exemplares. Este fato pode estar relacionado à migração desses espécimes para reprodução (BARTHEM \& FABRÉ, 2004), fato comprovado pela ausência de indivíduos adultos, observado pelo tamanho médio dos animais, dado que se capturaram exemplares jovens e pré-adultos. Por outro lado, a falta da captura de um maior número de exemplares pode também estar associada à busca por um local onde haja alimento, visto que as áreas, em especial II, III e IV, correspondem aos efluentes de resíduos sólidos e líquidos.

Nos ambientes aquáticos, a utilização de órgãos de animais para estudos histológicos informa o potencial de impacto em espécies nativas mediante a exposição por poluentes (DE LA TORRE et al., 2005). O fígado tem recebido uma atenção particular nos estudos toxicológicos, em virtude de seu papel central no metabolismo e sua sensibilidade a poluentes ambientais (ARELLANO et al., 1999). Não há diferença na estrutura hepática dos peixes teleósteos de ambiente marinho e de água doce (GONZÁLES et al., 1993; VICENTINI et al., 2005). No presente 
estudo, evidenciou-se um grau acentuado de alterações estruturais no fígado, determinado pela desorganização do parênquima e pelo peso hepático dos animais que variou entre as áreas de captura. Essas alterações foram determinadas pelo acúmulo de gordura, caracterizando a degeneração gordurosa, bem como pela presença de células sanguíneas no parênquima hepático, diagnosticada como hepatite e congestão, e por último pela inflamação evidenciada pela presença de vacuolização celular (HIBIYA, 1982). A presença de cobre pode levar a alterações como vacuolização e necrose no fígado de Oreochromis niloticus (FIGUEIREDOFERNANDES et al., 2007b).

Segundo ARELLANO et al. (1999), a exposição de Solea senegalensis em concentração de $100 \mu \mathrm{g}$ de $\mathrm{Cu}^{+2} / \mathrm{L}$ apresentou aumento de células sanguíneas nos sinusoides e vênulas hepáticas. Tal alteração foi também encontrada em peixes coletados em locais onde há intensa descarga de poluente doméstico, observados em Cyprinus carpio exposto ao herbicida controlador de algas em tanques de piscicultura, em Piaractus mesopotamicus expostos às diversas concentrações de um organofosforado utilizado para controle de espécies zooplanctônicas e vermes de peixes (NESKOVIC et al., 1996; GÜL et al., 2004; MATAQUEIRO, 2006). Processos patológicos semelhantes foram encontrados nos indivíduos capturados nas áreas II e III, áreas identificadas pelo aporte de efluentes domésticos.

O tempo de permanência a um agente estressor ou a um local impactado também provoca alterações histopatológicas nos órgãos de peixes. MALBROUK et al. (2003) evidenciaram que peixes juvenis da espécie Carassius auratus, ao serem expostos à concentração de $125 \mu \mathrm{g} / \mathrm{Kg}$ de uma hepatotoxina produzida por cianobactérias, apresentaram desorganização de estrutura celular com algumas regiões de inflamação no citoplasma em apenas oito horas de exposição. LANGIANO \& MARTINEZ (2004) verificaram que os fígados dos peixes que habitavam um córrego que recebiam efluentes do mais variados tipos apresentaram degeneração celular e vacuolização citoplasmática. Resultados semelhantes também foram obtidos no presente estudo.

O tipo de alimentação e a qualidade do alimento influenciam na estrutura do fígado e podem provocar lesões ao tecido hepático. CABALLERO et al. (1999), estudando Sparus aurata alimentados com rações com diferentes níveis de lipídio e proteínas, encontraram uma diferença no crescimento dos espécimes e alterações hepáticas, confirmando a presença de inflamação e de acúmulo de lipídios. Esses processos patológicos também foram observados nos fígados de B. rousseauxii capturados nas áreas II e III.

Os centros melanomacrófagos (CMMs) foram poucos evidenciados nos fígados do presente estudo. É relatado que as variações do tamanho e do número de CMMs podem estar relacionadas com a idade, o estado nutricional, a doença e as condições ambientais. O pigmento melanina pode ter um efeito protetor contra os poluentes, e níveis elevados de melanina e lipofucsina podem proteger contra danos celulares, pois absorvem radicais livres (HARTLEY et al., 1996). O fato de não se ter encontrado CMMs em abundância em todos os fígados analisados, especialmente os fígados alterados, sugere um dano tecidual agudo, comprometendo o mecanismo de defesa e podendo até levar à morte do peixe.

$\mathrm{Na}$ área controle, verificou-se que a quantidade de indivíduos que apresentaram fígado sem alterações hepáticas foi a mesma dos indivíduos que apresentaram fígados com o parênquima alterado. Esses dados levam a acreditar que há uma migração dos espécimes, visto que também foram encontrados nas outras áreas indivíduos com fígados normais, sendo em menor número. Pelo número reduzido de espécimes e pelos processos patológicos observados, sugere-se que a Baía do Guajará apresenta início de modificação ambiental e que medidas de controle devem ser iniciadas para minimizar esse processo.

\section{CONCLUSÕES}

Considerando-se o baixo número de exemplares capturados no período em estudo e a determinação de $70 \%$ de espécimes com fígados apresentando processos patológicos em todas as áreas de estudo, pode-se sugerir o comprometimento da Baía do Guajará e que a espécie $B$. rousseauxii provavelmente está adaptada às áreas onde foram registrados os lançamentos de poluidores ambientais. No entanto, ainda é necessário estudo que avalie a qualidade da água, a fim de se determinar a intensidade de alteração físico-químico na Baía do Guajará. 


\section{REFERÊNCIAS}

ARELLANO, J.M.; STORCH, V.; SARASQUETE, C. Histological changes and copper accumulation in liver and gills of the senegales sole solea senegalensis. Ecotoxicology and Environmental Safety, v. 44, n. 1, p. 62-72, 1999.

AU, D.W.T. The application of histo-cytopathological biomarkers in marine pollution monitoring: a review. Marine Pollution Bulletin, v. 48, n. 9-10, p. 817-834, 2004.

AYRES, M.; AYRES-Jr, M.; AYRES, D.L.; SANTOS, A.A.S. Bioestat: aplicações estatísticas nas áreas das Ciências Biomédicas. Versão 5.0. Belém, Pará: Sociedade Civil Mamirauá, MCT-CNPq, 2007. 324 p.

BOMBONATO, M.T.S.; ROCHEL, S.S.; VICENTINI, C.A.; VICENTINI, I.B.F. Estudo morfológico do tecido hepático de $\mathrm{Le}$ porinus macrocephalus. Acta Scientiarum - Biological Sciences, v. 29 , n. 1, p. $81-85,2007$.

BRUSLÈ, J.; ANADON, G.G. The structure and fuction of fish liver. In: MUNSHI, J.S.D.; DUTTA, H.M. (Ed.). Fish morphology horizon of new research. Beirute: Science Publisher Inc., 1996. $16 \mathrm{p}$.

CABALLERO, M.J.; LÓPEZ-CALERO, G.; SOCORRO, J.; ROO, F.J.; IZQUIERDO, M.S.; FERNANDEZ, A.J. Combined effect of lipid level and fish meal quality on liver histology of gilthead seabream (Sparus aurata). Aquaculture, v. 179, n. 1-4, p. 277-290, 1999 .

DE LA TORRE, F. R.; FERRARI, L.; SALIBIÁN, A. Biomarkers of a native fish species (Cnesterodon decemmaculatus) application to the water toxicity assessment of a peri-urban polluted river of Argentina. Chemosphere, v. 59, n. 4, p. 577-583, 2005.

FANTA, E.; RIOS, F.S.; ROMÃO, S.; VIANNA, A.C.C.; FREIBERGER, S. Histopathology of fish Corydoras paleatus contaminated with sublethal levels of organophosphorus in water and food. Ecotoxicology and Environmental Safety, v. 54, n. 2, p. 119-130, 2003.

FIGUEIREDO-FERNANDES, A.M.; FONTAIINHAS-FERNANDES, A.A.; MONTEIRO, R.A.F.; REIS-HENRIQUE, M.A.; ROCHA, E. Spacial relationships of the intrahepatic vascular-biliary tracts and associated pancreatic acini of Nile tilapia, Oreochromis niloticus (Teleostei, Cichlidae): a serial section study by light microscopy. Annals of Anatomy, v. 189, p. 17-30, 2007a.

FIGUEIREDO-FERNANDES, A.; FERREIRA-CARDOSO, J.V.; GARCIA-SANTOS, S.; MONTEIRO, S.M.; CARROLA, J.; MATOS, P.; FONTAINHAS-FERNANDES, A. Histopathologicalç changes in liver and gill epithelium of Nile tilapia, Oreochromis niloticus, exposed to waterborne copper. Pesquisa Veterinária Brasileira, v. 27, p. 103-109, 2007b.

GERNHÖFER, M.; PAWERT, M.; SCHRAMM, M.; MÜLLER, E.; TRIEBSKORN, R. Ultraestructural biomarkers as tools to characterize the health status of fish in contaminated streams. Journal of Aquatic Ecosystem Stress and Recovery, v. 8, n. 3-4, p. 241-260, 2001.

GONZALES, G.; CRESPO, S.; BRUSLÉ, J. Histo-cytological study of the liver of the cabrilla sea brass, Serranus cabrilla (Teleostei, Serranidae), an available model for marine fish experimental studies. Journal of Fish Biology, v. 43, n. 3, p. 363-373, 1993.

GUL, S.; BELGE-KURUTAS, E.; YILDIZA, E.; SAHANC, A.; DORAN, F. Pollution correlated modifications of liver antioxidant system and histopathology of fish (Cyprinidae) living in Seyhan Dam Lake, Turkey. Environmental International, v. 30, n. 5, p. 605-609, 2004.

HARTLEY, W.R.; THIYAGARAJAH, A.; TREINIES, A.M. Liver lesions in the Gar Fish (Lepisosteidae) as biomarkers of exposure. Marine Environmental Research, v. 42, n. 1-4, p. 217-221, 1996.

HIBIYA, T. An atlas of fish histology: normal and pathological features. New York: Kodansha Tokyo, 1982. 147 p.

LANGIANO, V. C.; MARTINEZ, C. B. R. Liver histopathology of feral freshwater fish populations collected along a contaminated stream. In: INTERNATIONAL CONGRESS ON THE BIOLOGY OF FISH, BEHAVIOUR, PHYSIOLOGY AND TOXICOLOGY INTERACTIONS IN FISH, 6., 2004, Manaus. Proceedings... Manaus, 2004. p. 241-245. Disponível em: http://www.fishbiologycongress.org/

LIVINGSTONE, D.R. Biotechnology and pollution monitoring: use of molecular biomarker in the aquatic environment. Journal of Chemical Technology \& Biotechnology, v. 57, n. 3, p. 195-211, 1993.

MALBROUCK, C.; TRAUSCH, G.; DEVOS, P.; KESTEMONT, P. Hepatic accumulation and effects of microcystin-LR on juvenile goldfish Carassius auratus L. Comparative Biochemistry and Physiology Part C, v. 135, n. 1, p. 39-48, 2003.

MATAQUEIRO, M. I. Alterações histopatológicas e determinação residual em pacu (Piaractus mesopotamicus HOLMBERG, 1887) após exposição ao triclorfom. 2006. 53 f. Tese (Doutorado) - Universidade Estadual Paulista, Centro de Aquicultura da UNESP, São Paulo, 2006.

NEŠKOVIĆ, N.K.; POLEKSIĆ, V.; ELEZOVIĆ, I.; KARAN, V.; BUDIMIR, M. Biochemical and histopathological effects of glyphosate on carp, Cyprinus carpio L. Bulletin of Environmental Contamination and Toxicology, v. 56, n. 2, p. 295-302, 1996.

PETRERE JR., M.; BARTHEM, R.B.; CÓRDOBA, E.A.; GÓMEZ, B.C. Review of the large catfish fisheries in the upper Amazon and the stock depletion of piraíba (Brachyplatystoma filamentosum Lichtenstein). Fish Biology and Fisheries, v. 14, n. 4, p. 403-414, 2004.

PINHEIRO, R.V.L. Estudo hidrodinâmico e sedimentológico do Estuário Guajará - Belém (PA). 1987. 163 f. Dissertação 
(Mestrado) - Universidade Federal do Pará, Centro de Geociências, Belém, Pará, 1987.

RUFFINO, M. L. Estatística pesqueira do Amazonas e Pará 2001. Manaus: Ibama; PróVárzea, 2002. 73 p.

RUFFINO, M. L. Estatística pesqueira do Amazonas e Pará 2002. Manaus: Ibama; PróVárzea, 2005. 84 p.

RUFFINO, M.L.; ISAAC, V.J. Ciclo de vida e parâmetros biológicos de algumas espécies de peixes da Amazônia brasileira. Recursos pesqueiros do Médio Amazonas: biologia e estatística pesqueira. Brasília: Instituto Brasileiro do Meio Ambiente e dos Recursos Naturais Renováveis, 2000. 350 p. (Coleção Meio Ambiente. Série Estudo Pesca, 22).
SILVA, D. F. Utilização de biomarcadores para avaliar a qualidade da água da Baía do Guajará e rio Guamá. 2006. 71 f. Dissertação (Mestrado) - Universidade Federal do Pará, Ciência Animal, Belém, Pará, 2006.

VICENTINI, C. A.; FRANCESCHINI-VICENTINI, I.B.; BOMBONATO, M.T.S.; BERTOLUCCI, B.; LIMA, S.G.; SANTOS, A.S. Morphological study of the liver in the Teleost Oreochromis niloticus. International Journal Morphology, v. 23, n. 3, p. 211-216, 2005.

WINKALER, E.U.; SILVA, A.G.; GALINDO, H.C.; MARTINEZ, C.B.R. Biomarcadores histológicos e fisiológicos para o monitoramento da saúde de peixes de ribeirões de Londrina, Estado do Paraná. Acta Scientiarum, v. 23, n. 2, p. 507-514, 2001.

Protocolado em: 20 dez. 2007. Aceito em: 3 jun. 2009. 